\title{
Glyphosate-Induced Encephalopathy: A Case Report
}

\author{
Ho Kyu Lee ${ }^{a}$ \\ Hyun Soo Park \\ Jung-Hwan $\mathrm{Oh}^{\mathrm{c}}$ \\ Jung Seok Lee ${ }^{\mathrm{c}}$ \\ a'Departments of Radiology, \\ ${ }^{\mathrm{b}}$ Emergency Medicine, and ${ }^{\mathrm{N}}$ Neurology, \\ Jeju National University \\ School of Medicine, Jeju, Korea
}

Dear Editor,

Glyphosate (N-phosphonomethyl glycine) is an organophosphorus compound that is commonly used as a broad-spectrum herbicide in Asian countries. ${ }^{1,2}$ There has recently been an increasing trend in the number of suicide attempts using glyphosate. ${ }^{1}$ Glyphosate intoxication presents clinically with gastrointestinal discomfort, liver or kidney dysfunction, cardiopulmonary instability, and altered consciousness. Around one-fifth of patients suffering from glyphosate intoxication reportedly present with altered consciousness, ${ }^{1}$ but few brain MRI findings for patients with glyphosate intoxication have been reported. ${ }^{3}$ Here we present a patient with bilateral hippocampal and fornical lesions after ingesting glyphosate herbicide.

A 34-year-old man was admitted to the hospital in a drowsy state with difficulty breathing and swallowing. He had ingested an unknown amount of glyphosate herbicide in a suicide attempt just prior to admission. On hospital day 1 he developed oliguria and metabolic acidosis with progressive renal failure. He underwent urgent hemodialysis, which markedly improved his clinical status. On hospital day 2 he was conscious and alert, but complained about memory problems. The findings of neurological examinations of the cranial nerves and upper and lower extremities were normal. Diffusion-weighted imaging (DWI) on hospital day 10 revealed hyperintense lesions in the bilateral hippocampi with corresponding hyperintense signals on the apparent diffusion coefficient (ADC) map, which is considered to be the T2-"shine-through" effect (Fig. 1A and B). T2-weighted imaging (T2WI) (Fig. 1C and D) and FLAIR imaging (Fig. 1E and F) revealed hyperintense lesions in the bilateral hippocampi and fornices with mild swelling.

We performed detailed neuropsychological tests on hospital day 12. The patient had received 14 years of education, and he scored 22/30 on the Mini Mental State Examination (MMSE), missing all three words on recall (0/3). Neuropsychological testing revealed impaired memory and executive function. Follow-up T2WI (Fig. 1G) and FLAIR imaging (Fig. $1 \mathrm{H}$ and I) performed 14 months later showed isosignal intensities in the bilateral hippocampi and fornices. Follow-up neuropsychological tests performed about 3 years later showed significant improvements in both memory and executive function, with him scoring 28/30 on the MMSE, missing one word on recall (2/3).

Brain MRI showed bilateral hippocampal and fornical lesions in the present case. These structures are an important part of the Papez circuit and are required for emotion and memory. ${ }^{4,5}$ There is only one previous report on the use of brain MRI to investigate glyphosate-induced encephalopathy, which revealed a small hyperintense lesion in the left hippocampus in DWI with a corresponding low-signal focus on the ADC map. ${ }^{3}$ Unlike the previous case showing a unilateral hippocampal infarction, ${ }^{3}$ brain MRI in our patient revealed hyperintense lesions in the bilateral hippocampi and fornices, and to the best of our knowledge their involvement in glyphosate intoxication has not been reported previously.

Previous studies have demonstrated that glutamate-mediated excitotoxicity might be one of the mechanisms underlying glyphosate-induced encephalopathy. ${ }^{2}$ Specifically, exposure to glyphosate increases calcium influx through NMDA receptors and voltage-dependent @ This is an Open Access article distributed under the terms of the Creative Commons Attribution Non-Commercial License (https://creativecommons.org/licenses/by-nc/4.0) which permits unrestricted non-commercial use, distribution, and reproduction in any medium, provided the original work is properly cited. 

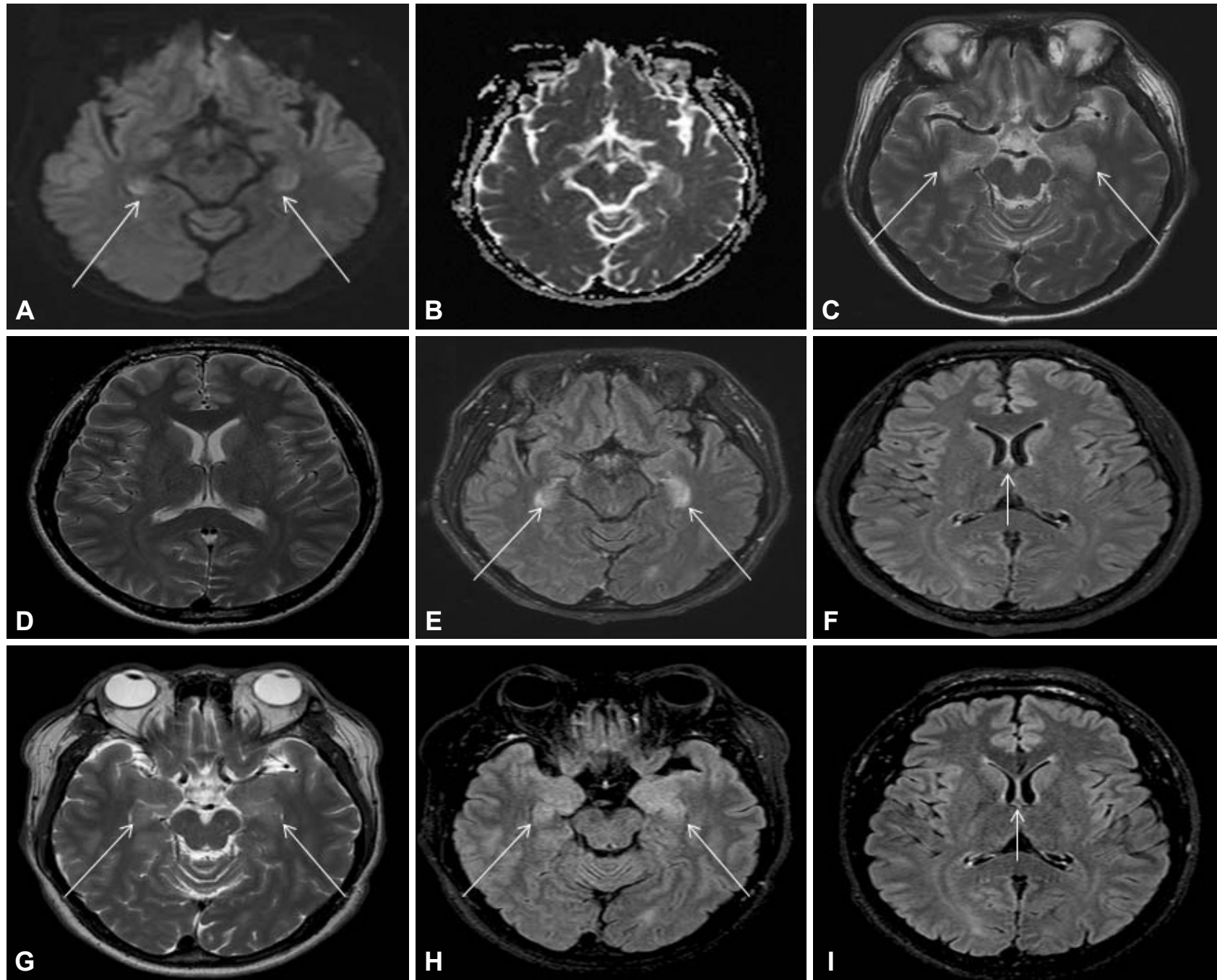

Fig. 1. Brain MRI scans of the patient. A-F: DWI performed 10 days after glyphosate intoxication shows hyperintense signals in the bilateral hippocampi (arrows, A). ADC map demonstrates slightly hyperintense signal in the adjacent brain tissues (B). T2WI images (arrows, C and D) and FLAIR images (arrows, E and F) show hyperintense signals in the bilateral hippocampi and fornices. G-I: Imaging performed 14 months after the initial MRI. T2WI images (arrows, G) and FLAIR images (arrows, H and I) show normalized signal intensities in the bilateral hippocampi and fornices. ADC: apparent diffusion coefficient, DWI: diffusion-weighted imaging, T2WI: T2-weighted imaging.

calcium channels, leading to neuronal cell death, especially in the hippocampus. ${ }^{2}$

The differential diagnosis of hippocampal hyperintense lesions in DWI includes acute ischemic stroke, transient global amnesia, seizure, limbic encephalitis, and hypoxic encephalopathy. ${ }^{6}$ Our patient developed encephalopathy after ingesting glyphosate herbicide, and this temporal relationship supports the diagnosis of glyphosate-induced encephalopathy.

In conclusion, the present case shows that glyphosate can cause reversible neurotoxicity to the hippocampus and fornix, and that brain DWI may be useful in detecting glyphosate-induced encephalopathy, especially in cases of memory impairment after glyphosate intoxication.

\section{Conflicts of Interest}

The authors have no financial conflicts of interest.

\section{REFERENCES}

1. Lee HL, Chen KW, Chi CH, Huang JJ, Tsai LM. Clinical presentations and prognostic factors of a glyphosate-surfactant herbicide intoxication: a review of 131 cases. Acad Emerg Med 2000;7:906-910.

2. Cattani D, Cesconetto PA, Tavares MK, Parisotto EB, De Oliveira PA, Reig CEH, et al. Developmental exposure to glyphosate-based herbicide and depressive-like behavior in adult offspring: implication of glutamate excitotoxicity and oxidative stress. Toxicology 2017;387:67-80.

3. Nishiyori Y, Nishida M, Shioda K, Suda S, Kato S. Unilateral hippocampal infarction associated with an attempted suicide: a case report. J Med Case Rep 2014;8:219.

4. Papez JW. A proposed mechanism of emotion. Arch Neurol Psychiat 1937:38:725-743.

5. Park SA, Hahn JH, Kim JI, Na DL, Huh K. Memory deficits after bilateral anterior fornix infarction. Neurology 2000:54:1379-1382.

6. Förster A, Griebe M, Gass A, Kern R, Hennerici MG, Szabo K. Diffusion-weighted imaging for the differential diagnosis of disorders affecting the hippocampus. Cerebrovasc Dis 2012;33:104-115. 